\title{
Current Topics in DØ B-Physics
}

\author{
Arthur Maciel ${ }^{a}$ * \\ ${ }^{a}$ Physics Department, Northern Illinois University, \\ DeKalb, Illinois, 60115-2870 USA
}

An overview of selected topics in DØ B-Physics is presented, covering relevant detector characteristics, and with emphasis on the most recent results in the $B_{s}$, FCNC and rare decay programs.

\section{INTRODUCTION}

The Tevatron, Fermilab's $\bar{p} p 1.96 \mathrm{GeV} / \mathrm{c}^{2}$ C.M. Collider, has recently reached a delivered total of $1 \mathrm{fb}^{-1}$ of integrated luminosity to its two multi-purpose detectors, $\mathrm{D} \varnothing$ and $\mathrm{CDF}$, during the current data taking period known as RunII. Initial difficulties with beam stability were efficiently corrected during a shutdown in late 2003, and since then the Tevatron complex has operated remarkably well, with impressive recovery during 2004 and matching its luminosity design goals in 2005 [1. The $\mathrm{D} \varnothing$ detector has been steadily accumulating good quality data throughout RunII with luminosity recording efficiencies now approaching the $90 \%$ mark [2].

This article presents an overview of selected D $\varnothing$ studies in B Physics, with emphasis on the most recent results, many of which are still preliminary. Specific analyses are covered in more detail elsewhere in these proceedings.

\section{THE DØ DETECTOR}

DØ's central magnetic tracking volume is a key ingredient to the B Physics program. Inexistent during RunI, it is a RunII upgrade addition and consists of a silicon microstrip tracker (SMT) surrounded by a central fiber tracker (CFT), both inside a $2 \mathrm{~T}$ superconducting solenoidal magnet. Optimized for tracking and vertexing within $|\eta|<3$, the SMT has a six barrel longitudinal structure, each with four layers of sensors. Barrels are interspersed with twelve F-disks (double

\footnotetext{
*For the DØ Collaboration
}

sided, $\pm 15^{\circ}$ stereo), with four extra $\mathrm{H}$-disks (single sided, $\pm 7.5^{\circ}$ stereo) located beyond the barrels to complete the $\eta$ coverage. The CFT covers tracking within $|\eta|<1.6$, and has eight thin coaxial cylindrical layers, each supporting two doublets of overlapping scintillating fibers arranged either longitudinally along $z$ or in stereo pairs. Both the CFT and the SMT provide tracking information to the trigger.

Outside the coil the next two layers of detection consist of a preshower (scintillator strips) and a liquid argon calorimeter. There follows an outer muon system, another key ingredient to DØ's B Physics program because of its ability to efficiently trigger on semi-muonic $b$ decays (or dimuons from $J / \psi$ etc), thus providing a remarkable selection tool for $b$ events in the hostile $\bar{p} p$ collider environment. The muon system covers $|\eta|<2$ and consists of a layer of multiple wire tracking devices and scintillation counters in front of $1.8 \mathrm{~T}$ iron toroids, followed by another two similar detection layers outside the toroids. A detailed description of the $D \varnothing$ detector can be found in 3 .

Every analysis presented here has one or more muons in its final state. This illustrates the importance of muons in the $\mathrm{D} \varnothing$ collection of a $\mathrm{B}$ enriched sample. Trigger decisions are organized in a tiered system with three levels of event inspection. Levels 1 and $2 \mathrm{~B}$ triggers are typically seeded by muon hits and complemented by tracking information. Level 1 muon candidates are about $60 \%$ pure, and can be further purified at Level 2 with adjustable time gates and/or 3D 
track matches. The CFT provides a fast and continuous readout to the Level 1 while the SMT provides signals to the levels 2 and 3 [4 for the detection of displaced vertices from $b$-quark decays. Level 3 receives slower more complete signals from all subdetectors enabling total reconstruction of the event, with the ability for accurate tracking, vertexing and invariant masses. Examples of B hadron reconstruction yields from the $\mathrm{D} \varnothing J / \psi$ dimuon triggers are given in table 1

Table 1

Number of decays reconstructed per $p b^{-1}$.

\begin{tabular}{lc}
\hline decay & $\sim N / p b^{-1}$ \\
\hline$B_{u}^{ \pm} \rightarrow J / \psi+K^{ \pm}$ & 21 \\
$B_{d}^{0} \rightarrow J / \psi+K^{*}(K \pi)$ & 8 \\
$B_{d}^{0} \rightarrow J / \psi+K_{s}^{0}(\pi \pi)$ & 2 \\
$B_{s}^{0} \rightarrow J / \psi+\phi(K K)$ & 2 \\
$\Lambda_{b}^{0} \rightarrow J / \psi+\Lambda^{0}(p \pi)$ & 0.3 \\
\hline
\end{tabular}

\section{THE $B_{s}$ PROGRAM}

One of the primary goals of RunII flavor physics at the Tevatron is the observation of $B_{s}$ mixing and the extraction of $\Delta m_{s}$. As part of its general effort in this direction, D $\varnothing$ presents in this conference two complementary analyses of the $B_{s}$ system; (i) a preliminary measurement of $\Delta \Gamma_{s} / \Gamma_{s}[\underline{5}$ and (ii) an exercise towards the measurement of $\Delta m_{s} \underline{\underline{6}}$.

For access to the relative width difference between $B_{s}$ mass eigenstates $\left(\Delta \Gamma_{s} / \Gamma_{s}\right)$ the decays $B_{s} \rightarrow J / \psi \phi$ are selected due to their non-flavorspecific final state, common to both meson and anti-meson decays. Assuming the two $B_{s}$ mass eigenstates correspond to the $\mathrm{CP}$ eigenstates, their components in the end products are separated by means of a simultaneous study of the $B_{s}$ time evolution, and the angular distribution of the final state particles [7]. $J / \psi \phi$ states in a $\mathrm{P}(\mathrm{S}$ or $\mathrm{D})$ wave indicate decays of the $\mathrm{CP}$ odd (even) eigenstates. A transversity angular variable particularly sensitive to this discrimina- tion is defined. An unbinned maximum likelihood fit to the data (about $450 p b^{-1}$ ) is performed, that combines the candidate $B_{s}$ mass, lifetime and transversity. Three parameters are extracted; (i) $f_{\text {odd }}^{(t=0)}$, the relative rate of the CPodd states at time zero, (ii) $\bar{\tau}=1 / \bar{\Gamma}$ where $\bar{\Gamma} \equiv\left(\Gamma_{\text {even }}+\Gamma_{\text {odd }}\right) / 2$, and (iii) $\Delta \Gamma / \bar{\Gamma}$. The resulting values are shown in table 2

Table 2

Results from the combined fit.

\begin{tabular}{cc}
\hline parameter & value \\
\hline $\bar{\tau}$ & $1.39_{-0.14}^{+0.13} \pm 0.08 p s$ \\
$\Delta \Gamma / \bar{\Gamma}$ & $0.21_{-0.40}^{+0.27} \pm 0.20$ \\
$f_{\text {odd }}^{(t=0)}$ & $0.17 \pm 0.10 \pm 0.02$ \\
\hline
\end{tabular}

Figure 1] shows the one sigma contours (stat and stat+syst) for $c \bar{\tau}$ versus $\Delta \Gamma / \bar{\Gamma}$, overlaid with the result from CDF 58 and the Standard Model prediction [9].

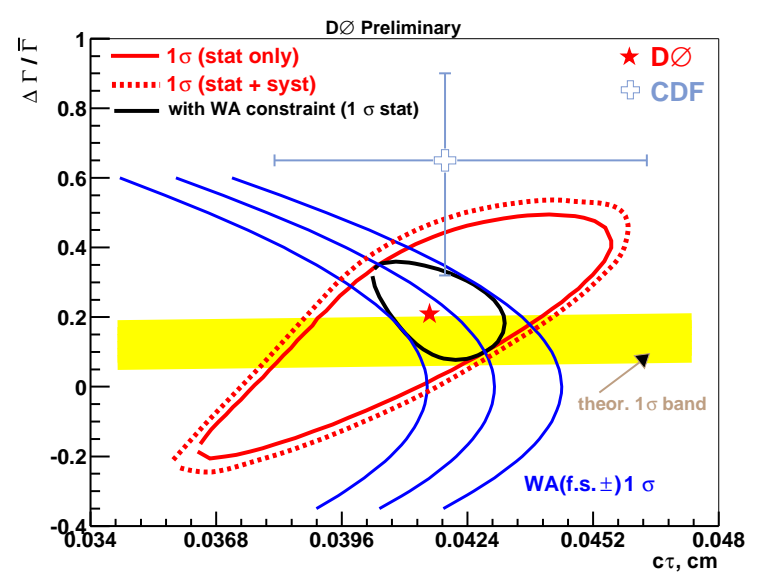

Figure 1. $1 \sigma$ contours for $c \bar{\tau}$ versus $\Delta \Gamma / \bar{\Gamma}$.

A suite of single muon triggers offers access to a large sample of semileptonic $B_{s}$ decays. With these, $\mathrm{D} \varnothing$ has recently completed what is currently the single most precise measurement of the 
$B_{s}$ lifetime [10. About $5000 B_{s} \rightarrow D_{s} \mu X$ decay candidates were selected from $400 p b^{-1}$ of data, where the $D_{s}$ is reconstructed from its $\phi \pi$ final state. Ignoring lifetime differences (i.e. assuming the simple $e^{-t / \tau}$ as functional form) the value $\tau\left(B_{s}\right)=1.420 \pm 0.043 \pm 0.057 \mathrm{ps}$ is obtained, and is compared with previous measurements and the revised world average [1] in figure 2]

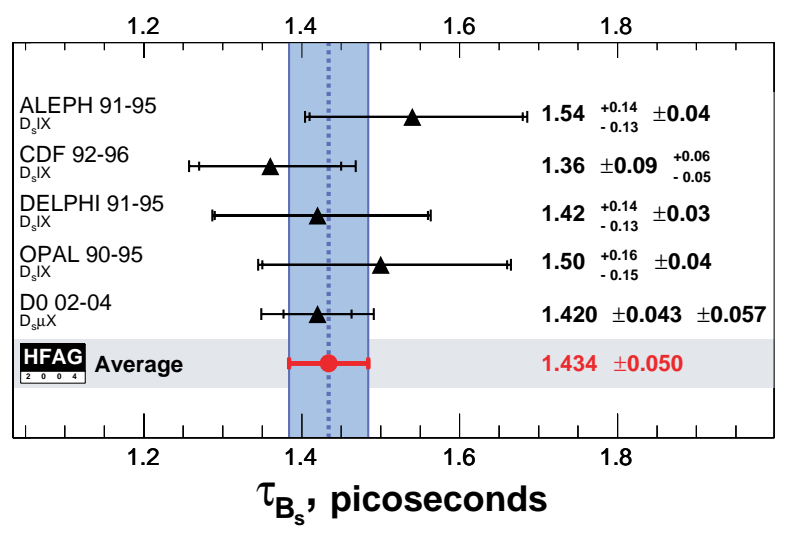

Figure 2. Individual $\tau\left(B_{s}\right)$ measurements and the world average [11].

This measurement of $\tau\left(B_{s}\right)$ provides an independent constraint between the $c \bar{\tau}$ and $\Delta \Gamma / \bar{\Gamma}$ of figure 1(since both results are extracted from different data samples) and therefore can be used to further reduce the $1 \sigma$ contours in that figure. This effect is represented in figure 1 by the three parabolas which map the uncertainty band of the WA value of figure 2 into the $c \bar{\tau}$ versus $\Delta \Gamma / \bar{\Gamma}$ plane. The smaller (inner) contour then represents the $\mathrm{D} \emptyset$ results now under these tighter constraints.

The large $B_{s} \rightarrow D_{s} \mu X$ decay sample, from which $\tau\left(B_{s}\right)$ is extracted, was also used in an exercise at setting a lower limit to $\Delta m_{s}$, aimed at developing the tools and methods for this measurement which still remains critically dependent on the accumulation of luminosity. The initial state flavor tagger builds a $b / \bar{b}$ likelihood from a choice of opposite side discriminating variables, and 380 fully tagged events are selected in the visible proper decay length range of $(-100,600) \mu \mathrm{m}$, from which the limit $\Delta m_{s}>5 p s^{-1}$ is extracted at $95 \%$ CL. This analysis is described in 612 .

Since this conference, an updated version of the $\Delta m_{s}$ analysis has been released [13]. Some upgrade features are improved selection methods and flavor taggers, plus an integrated luminosity of $610 p b^{-1} . D_{s} \rightarrow K K^{*}$ is an added contribution, and soft electron tagging is used for the first time in $\mathrm{D} \varnothing$, in combination with other taggers. The currrent result is $\Delta m_{s}>7.3 p s^{-1}$ at $95 \%$ CL, and indicates the pace at which this project progresses. Large areas of improvement still remain untapped, covering analysis techniques (added channels, same side tagging, unbinned fits) as well as the use of hadronic $B_{s}$ decays.

\section{FCNC, RARE DECAYS}

$\mathrm{D} \varnothing$ has search programs for $f c n c$ decays in both $B$ and $C$ sectors. Forbidden at tree level in the Standard Model (SM), these decays must proceed via higher order $W I$ processes. Such suppression allows for physics beyond the SM, such as large $\tan \beta$ SUSY scenarios with an extended Higgs sector, to become competitive with, or even dominant over the SM itself.

In the $B$ sector, $\mathrm{D} \varnothing$ 's upper limit to $B R\left(B_{s} \rightarrow\right.$ $\mu^{+} \mu^{-}$) 14] has recently been updated [15] for additional integrated luminosity, and set at $B R<$ $3.7 \cdot 10^{-7}(95 \% \mathrm{CL})$, still two orders of magnitude above SM expectations. A parallel analysis towards setting an upper limit to $B R\left(B_{s} \rightarrow\right.$ $\left.\mu^{+} \mu^{-} \phi\right)$ was work in progress at the time of this conference. Its result has recently been released [16] at $B R<4.1 \cdot 10^{-6}(95 \% \mathrm{CL})$, now approaching the SM predicted [17] central value of $1.6 \cdot 10^{-6}$.

The $C$ sector is a rather different (and comparatively untapped) testing ground for new phenomena. While the $B$ sector has top-assisted higher order loops, $C$ sector fcnc transitions involve loops with lighter fermions and have a more efficient GIM cancellation. SM extensions may upset this cancellation, and a sensitive observable [18 to which $\mathrm{D} \varnothing$ has efficient access (via dimuon triggers) is the differential decay rate 
$\mathcal{R}=d \Gamma\left(\mu^{+} \mu^{-} \pi\right) / d m\left(\mu^{+} \mu^{-}\right)$. D $\varnothing$ has started a program of searches for excess counts in $\mathcal{R}$, in the continuum regions of the $\mu^{+} \mu^{-} \pi$ spectrum. To this end, a first step has been a study of resonant regions for setting normalization benchmarks. With $508 p b^{-1}$ of analyzed data, DØ observes the $D_{s}^{+} \rightarrow \phi \pi^{+} \rightarrow \mu^{+} \mu^{-} \pi^{+}$final state with a significance above background greater than $7 \sigma$ 19. The signal is shown in figure [3] where a neighboring peak suggests a $2.7 \sigma$ signal for the $D^{+} \rightarrow \phi \pi^{+} \rightarrow \mu^{+} \mu^{-} \pi^{+}$decay, from which a preliminary (and best existing) limit for this decay is set at $B R<3.1 \cdot 10^{-6}(90 \% \mathrm{CL})$.

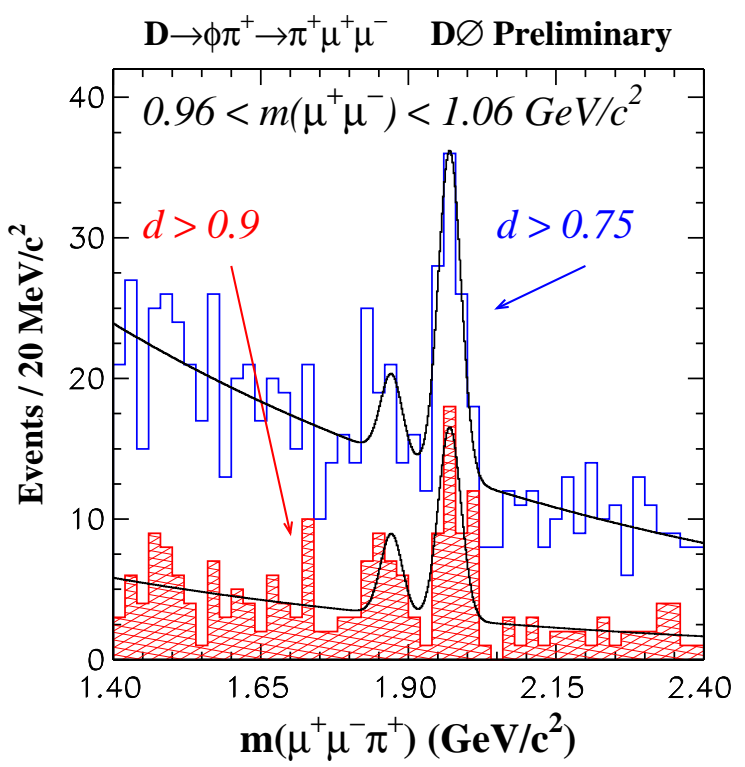

Figure 3. The $\mu^{+} \mu^{-} \pi$ mass spectrum for two values of a likelihood variable $d$.

\section{CONCLUSION}

$\mathrm{D} \varnothing$ is producing a wealth of heavy flavor results in RunII, with significant impact on world averages and limits. Key measurements such as $B_{s}$ mixing are on track, with uncertainty dominated by statistics, not systematics. Beyond the studies presented here, there are results in spectroscopy, production, baryons, lifetimes etc, some of which were covered in this conference 20 . In the near future, a Tevatron shutdown is scheduled for three months, when $\mathrm{D} \varnothing$ will undergo trigger upgrades, with an extra $50 \mathrm{~Hz}$ of bandwidth to tape dedicated to flavor physics. An inner layer (Layer-0) will be added to the silicon detector.

\section{REFERENCES}

1. Tevatron luminosity plots can be viewed at http://www-bdnew.fnal.gov/pbar/AEMPlots /today/DataSummaryTables.html

2. $\mathrm{D} \varnothing$ luminosity plots can be viewed at http://www-d0.fnal.gov/runcoor/RUN

/run2_lumi.html

3. "The Upgraded DØ Detector" submitted to Nuclear Instruments and Methods in Physics Research A (NIM-A), Fermilab-Pub-05-341$\mathrm{E}$, or on the arXiv as physics/0507191.

4. "The STT Trigger at DØ", Sasha Caron, these proceedings.

5. " $\Delta \Gamma_{s} / \Gamma_{s}$ at the Tevatron" Alberto Sanchez, these proceedings.

6. " $B_{d}$ and $B_{s}$ Mixing at DØ", Tania Moulik, these proceedings.

7. "Measurement of the lifetime difference in the $B_{s}$ system", hep-ex/0507084, and accepted by PRL (in print).

8. CDF Collaboration, PRL 94, 101803 (2005).

9. I.Dunietz, R.Fleisher and U.Nierste, PRD 63, 114015 (2001).

10. DØ note 4729-CONF.

11. Heavy Flavor Ave. Group, hep-ex/0505100, http://www.slac.stanford.edu/xorg/hfag/

12. DØ note 4724-CONF.

13. DØ note $4878-\mathrm{CONF}$.

14. DØ Collaboration, PRL 94, 071801 (2005).

15. "Rare Decays at the Tevatron", S. Farrington, these proceedings, and DØnote 4733-CONF.

16. DØnote 4862-CONF.

17. C.Geng and C.Liu, J.Phys.G 29, 1103 (2003).

18. G. Burdman et.al., PRD 66, 014009 (2002).

19. DØnote 4731-CONF.

20. In these proceedings, reports by T. Moulik, S. Farrington, A. Sanchez, D. Bauer, W. Wester, E. de la Cruz-Burelo, U. Kerzel. 